\title{
Comparison of 1-year outcomes between MAKO versus NAVIO robot-assisted medial UKA: nonrandomized, prospective, comparative study
}

\author{
Chumroonkiet Leelasestaporn', Tomorn Tarnpichprasert ${ }^{1}$, Alisara Arirachakaran ${ }^{2}$ and Jatupon Kongtharvonskul ${ }^{3,4^{*}}$
}

\begin{abstract}
Background: We have conducted a prospective cohort study with the aim of comparing operative time, intraoperative blood loss, and radiologic and clinical outcomes between imageless (NAVIO) and image-based (MAKO) robot-assisted unicompartmental knee arthroplasty (UKA) for medial compartment osteoarthritis (OA) of the knee.

Methods: A total of 33 patients with medial compartment OA of the knee were prospectively allocated on alternate operative days of their surgery to MAKO (16 patients) or NAVIO (17 patients) robot-assisted UKA. The primary outcome (Knee Society Score [KSS] and Knee Functional Score [KFS]) and the secondary outcomes (intraoperative time of seven steps [registration of hip and ankle, femur and tibia, ligament tension, implant planning, preparation femur, tibia and trial implant], component alignment [coronal and sagittal of femur, tibia implant], blood loss, complications, and revision at 1 year after surgery) were compared between two groups. Statistical significance was set at $P<0.05$.
\end{abstract}

Results: Mean KSS measured at baseline and 1 year were, respectively, 70.3 (5.6) and 96.9 (5.7) in the NAVIO group and 72.3 (4.5) and 94.7 (10.01) in the MAKO group. Mean KFS measured at baseline and 1 year were, respectively, 95.5 (7.9) and 99.9 (0.25) in the NAVIO group and 67.3 (7.8) and 99.5 (1.2) in the MAKO group. There were no significant differences for KFS and KSS outcomes $(P=0.203$ and $P=0.457$, respectively) between the NAVIO and MAKO groups. Mean operative time and blood loss in the NAVIO versus MAKO robot-assisted UKA groups were 98 min versus $82.5 \mathrm{~min}$ and $136.3 \mathrm{ml}$ versus $80 \mathrm{ml}$, respectively, and these differences were statistically significant. In the MAKO group, the intraoperative time was statistically significantly shorter in registration of hip and ankle center, femur and tibia, femur preparation, and trial implantation compared with the NAVIO group. There were no significant differences of component alignment and radiologic alignment at 1 year between the two groups. No perioperative or delayed complications (infection, periprosthetic fracture, thromboembolism, and compromised wound healing) and revisions were reported in either group.

Conclusions: This study demonstrated that two robotic systems showed no difference in clinical outcomes at 1 year and radiologic alignment of implants, whereas operative time and intraoperative blood loss were found to be less in MAKO robot-assisted UKA.

Trial registration: ClinicalTrials.gov, NCT03954912. Registered on 17 May 2019.

(Continued on next page)

\footnotetext{
* Correspondence: Jatupon_kong@hotmail.com

${ }^{3}$ Section for Clinical Epidemiology and Biostatistics, Faculty of Medicine,

Ramathibodi Hospital, Bangkok, Thailand

${ }^{4}$ Orthopedic Department, Payathai 3 Hospital, Bangkok, Thailand

Full list of author information is available at the end of the article
}

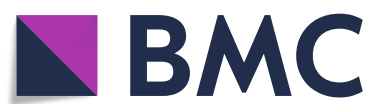

Part of Springer Nature (c) The Author(s). 2020 Open Access This article is distributed under the terms of the Creative Commons Attribution 4.0 International License (http://creativecommons.org/licenses/by/4.0/), which permits unrestricted use, distribution, and reproduction in any medium, provided you give appropriate credit to the original author(s) and the source, provide a link to the Creative Commons license, and indicate if changes were made. The Creative Commons Public Domain Dedication waiver (http://creativecommons.org/publicdomain/zero/1.0/) applies to the data made available in this article, unless otherwise stated. 
(Continued from previous page)

Level of evidence: ॥

Keywords: MAKO, NAVIO, Robotic surgery, UKA

\section{Introduction}

Unicompartmental knee arthroplasty (UKA) offers potential functional advantages over total knee arthroplasty (TKA) $[1,2]$. One of the greatest challenges to both uptake of UKA by surgeons and the ultimate success of the surgery has been the technically demanding nature of the surgery. Recent changes in component design, surgical instrumentation, and surgical techniques have led to improved UKA radiographic and clinical outcomes of UKA [3-6]. The changes in surgical instruments that have taken place include systems that allow more accurate flexion-extension gap balancing and more accurate bone preparation. However, despite these improvements in manual instruments, some surgeons have also recently adopted advances in robotic surgery that have led to improved accuracy and alignment of UKA prostheses [613]. Currently, there are two semiautonomous systems approved by the U.S. Food and Drug Administration for robot-assisted UKA: an imageless (NAVIO) system [14] and an image-based (MAKO) system $[6,8,13,15,16]$. The current semiautonomous systems use different methods to safeguard against inadvertent bone preparation, one by providing haptic constraint beyond which movement of the burr is limited (MAKO: Restoris MCK partial knee implant system; Stryker, Kalamazoo, MI, USA) and the other by modulating the exposure or speed of the handheld robotic burr (NAVIO: Journey UNI unicompartmental knee system; Smith \& Nephew, Memphis, TN, USA). These systems also provide realtime quantification of soft-tissue balancing, which may contribute to the reported successful clinical and functional outcomes with semiautonomous systems [17]. The results show that both robot-assisted surgical systems have better intraoperative (surgical time and blood loss) and postoperative (range of motion [ROM], function, complications, and revisions) outcomes and return to activity than conventional UKA [13-16]. However, no randomized controlled trials, systematic reviews, or meta-analyses have compared intraoperative (surgical time, tourniquet time, operative time and blood loss) and postoperative (ROM, function, complications, revisions and return to activity) outcomes of the NAVIO versus MAKO systems in UKA. Therefore, we conducted a prospective cohort study with the aim of comparing operative time, intraoperative blood loss, and radiologic and clinical outcomes between imageless (NAVIO) and image-based (MAKO) robot-assisted UKA for medial compartment osteoarthritis (OA) of the knee.
We hypothesized that use of the MAKO system in medial UKA would improve clinical outcomes, including intraoperative (surgical time and blood loss) and postoperative outcomes, compared with the NAVIO system.

\section{Methods}

Our study included 33 patients who underwent medial UKA performed by a single senior surgeon (CL) with either the NAVIO or MAKO robot-assisted system between 1 June 2015 and 1 July 2018 at Bhumibol Adulyadej Hospital. This study was approved by the Committee on Human Rights Related to Research Involving Human Subjects at the Bhumibol Adulyadej Hospital under the protocol ID 2/62. All patients had been listed for UKA to treat medial compartment knee OA and were recruited by a research associate (TT). Eligible patients were those deemed suitable for UKA by a senior surgical author (CL), could give informed consent, and were willing to attend the prescribed followup. Exclusion criteria included those with ligament insufficiency (anterior cruciate ligament rupture; collateral ligament insufficiency); inflammatory arthritis; a deformity requiring augmentation; neurological movement disorders; pathology of the feet, ankles, hips, or opposite knee causing significant pain or gait alterations; and those who ultimately required TKA. (Valgus greater than 14 degrees and multiple compartment OA were contraindications to UKA.) The 33 patients were allocated for either image-based (MAKO) or imageless (NAVIO) procedures on alternate days of their surgery, with 16 assigned to the NAVIO robot-assisted UKA cohort and 17 to the MAKO robot-assisted UKA cohort (Fig. 1). Four UKA procedures were performed regularly in 1 day. There were no significant demographic differences between the groups (Table 1).

\section{Surgical technique}

All surgical procedures were performed by the same surgeon (CL) with the patient under either epidural or general anesthesia. A tourniquet was applied and inflated before registration. All patients received $1 \mathrm{~g}$ of tranexamic acid at induction. Patients in both treatment groups received a combination of $20 \mathrm{ml}$ of $0.5 \%$ bupivacaine, normal saline solution $40 \mathrm{ml}$, Ketorolac $30 \mathrm{mg}$ (Pfizer, New York, NY, USA), and adrenaline $0.5 \mathrm{ml}$ injected into the joint capsule prior to wound closure. The tourniquet was deflated after wound closure. The implant used was a 


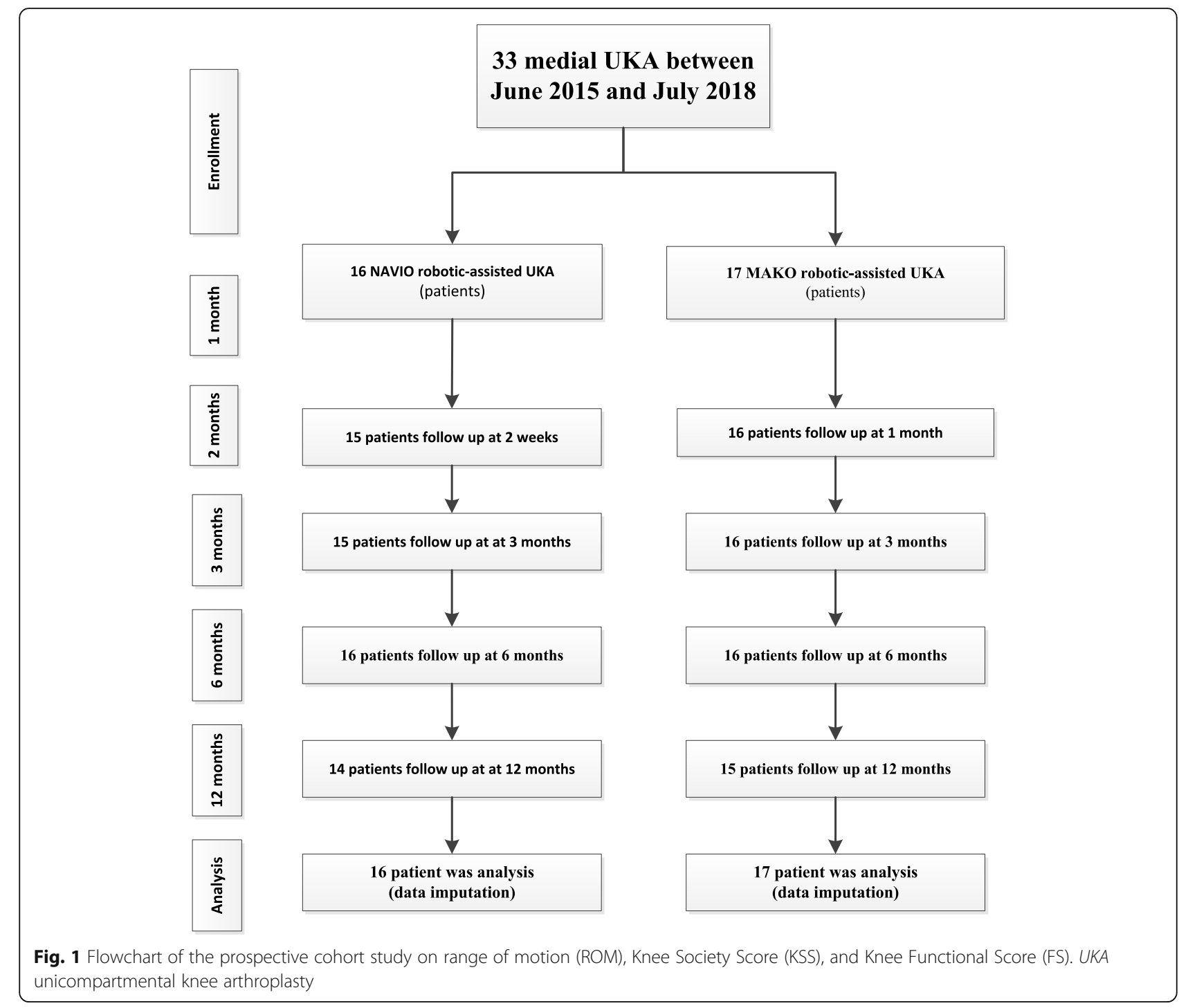

cemented, fixed-bearing unicompartmental prosthesis with metal-bearing polyethylene (Restoris MCK partial knee implant system; Stryker) in the image-based MAKO robotic system and the Journey UNI Unicompartmental knee system (Smith \& Nephew) in the imageless NAVIO system. In the MAKO group, preoperative computed tomography was performed and a 3D computer model of the knee was constructed by a trained technician. A medial parapatellar quadriceps-sparing incision and approach were used, and UKA was performed using the instrumentation in accordance with the operative technique. All three articular compartments and the cruciate ligaments were examined to confirm suitability for UKA. The proper surgical technique of image-based MAKO [15] and imageless NAVIO [18] robot-assisted UKA have been described previously. Figure 2 shows postoperative $\mathrm{x}$-rays of $\mathrm{MAKO}$ and NAVIO robotassisted UKA.

\section{Postoperative rehabilitation}

All patients in both groups followed the same standardized postoperative rehabilitation program, with full weight-bearing and active ROM exercises commenced from the day of surgery. Each physiotherapy session lasted $25 \mathrm{~min}$ in total, and all rehabilitation was performed by the same multidisciplinary team in both treatment groups. Patients were discharged after adequate pain control, knee flexion to a minimum of 90 degrees, independent mobilization with the use of crutches, and independent ascent and descent of stairs. All study patients were discharged to home. No patients were discharged to a rehabilitation center or other skilled nursing facility.

\section{Clinical assessment}

Preoperative data regarding age, sex, body mass index, and ROM were recorded. Patients were followed up 
Table 1 Characteristics of patients who underwent NAVIO and MAKO robotically assisted unicompartmental knee arthroplasty at baseline, intraoperatively, and 1 year postoperatively

\begin{tabular}{|c|c|c|c|}
\hline Outcomes & $\begin{array}{l}\text { NAVIO robotically assisted UKA } \\
(n=16)\end{array}$ & $\begin{array}{l}\text { MAKO robotically assisted UKA } \\
(n=17)\end{array}$ & $P$ value \\
\hline \multicolumn{4}{|l|}{ Baseline characteristics } \\
\hline Age, yr, mean (SD) & $70.9(5.9)$ & $71.5(6.3)$ & 0.573 \\
\hline \multicolumn{4}{|l|}{ Sex, $n(\%)$} \\
\hline Male & $4(25.0)$ & $4(23.5)$ & 0.922 \\
\hline Female & $12(75.0)$ & $13(76.5)$ & \\
\hline Weight, kg, mean (SD) & $64.8(9.5)$ & $62.4(9.3)$ & 0.479 \\
\hline Height, cm, mean (SD) & $157.8(7.0)$ & $155.1(4.8)$ & 0.203 \\
\hline $\mathrm{BMI}, \mathrm{kg} / \mathrm{m}^{2}$, mean (SD) & $26.0(3.17)$ & $25.8(3.3)$ & 0.912 \\
\hline Preoperative ROM in flexion, degrees, mean (SD) & $130(5.5)$ & $129.4(5.8)$ & 0.768 \\
\hline Preoperative ROM in extension, degrees, median (range) & $0(0-5)$ & $0(0)$ & 0.07 \\
\hline Preoperative KSS, mean (SD) & $70.3(5.6)$ & $72.3(4.5)$ & 0.265 \\
\hline Preoperative KFS, mean (SD) & $65.5(7.9)$ & $67.3(7.8)$ & 0.515 \\
\hline \multicolumn{4}{|l|}{ Intraoperative results } \\
\hline \multicolumn{4}{|l|}{ Method of anesthesia } \\
\hline Epidural anesthesia (\%) & $14(87.5)$ & $16(0.94)$ & 0.509 \\
\hline General anesthesia (\%) & $2(12.5)$ & $1(0.06)$ & \\
\hline Tourniquet time, minutes, mean (SD) & $113.6(8.4)$ & $109.4(18.7)$ & 0.416 \\
\hline All seven surgical step time, min, mean (SD) & $98.0(8.4)$ & $82.5(11.8)$ & 0.0002 \\
\hline Operative time, min, mean (SD) & $134.4(9.3)$ & $130.4(20)$ & 0.472 \\
\hline Blood loss, ml, median (range) & $136.3(108-155)$ & $80(68-132)$ & 0.006 \\
\hline \multicolumn{4}{|l|}{ Postoperative results } \\
\hline Hospital stay, d, mean (SD) & $4.8(1.2)$ & $4.4(1.1)$ & 0.406 \\
\hline \multicolumn{4}{|l|}{ Radiographic postoperative findings } \\
\hline Acceptable femur varus/valgus angle & 16 & 17 & - \\
\hline Acceptable femur flexion/extension angle & 16 & 17 & - \\
\hline Acceptable tibia varus/valgus angle & 16 & 17 & - \\
\hline Acceptable tibia posteroinferior angle & 16 & 17 & - \\
\hline ROM in flexion at $1 \mathrm{yr}$, degrees, mean (SD) & $130.3(5.6)$ & $131.2(6.7)$ & 0.693 \\
\hline ROM in extension at $1 \mathrm{yr}$, degrees, mean (SD) & $0(0)$ & $0(0)$ & - \\
\hline Knee Functional Score at 1 yr, mean (SD) & $99.9(0.25)$ & $99.5(1.2)$ & 0.203 \\
\hline Knee Society Score at $1 \mathrm{yr}$, mean (SD) & $96.9(5.7)$ & $94.7(10.1)$ & 0.457 \\
\hline Postoperative complications at $1 \mathrm{yr}$ & 0 & 0 & - \\
\hline Revisions at $1 \mathrm{yr}$ & 0 & 0 & - \\
\hline
\end{tabular}

Acceptable femur varus/valgus angle of the femoral component relative to the femur is $<10$ degrees varus to $<10$ degrees valgus)

Acceptable femur flexion/extension angle of the femoral component relative to the femur is within 15-degree flexion to 0-degree extension

Acceptable tibia varus/valgus angle of the tibial component relative to the tibia is $<5$ degrees varus to $<5$ degrees valgus

Acceptable tibia posteroinferior tilt of the tibial component relative to the tibia is $7 \pm 5$ degrees

Abbreviations: BMI body mass index, KFS Knee Functional Score, KSS Knee Society Score, ROM range of motion, UKA unicompartmental knee arthroplasty

postoperatively at 12 months. Full hospital and clinic medical record review of demographic, preoperative, intraoperative (mean all 7 surgical step time and tourniquet time), intraoperative blood loss (visible blood loss were observed before tourniquet release), and postoperative knee score measurements (Knee Functional Score [KFS] and Knee Society Score [KSS]) was performed. The primary outcomes of interest were KSS and KFS. Mean intraoperative time was divided into seven steps (registration of hip and ankle, femur and tibia, ligament tension, implant planning, preparation femur, tibia, and trial implant). The KSS questionnaire includes seven items and has a maximum score of 100 (the higher the score, the better the function), and the KFS questionnaire includes four 


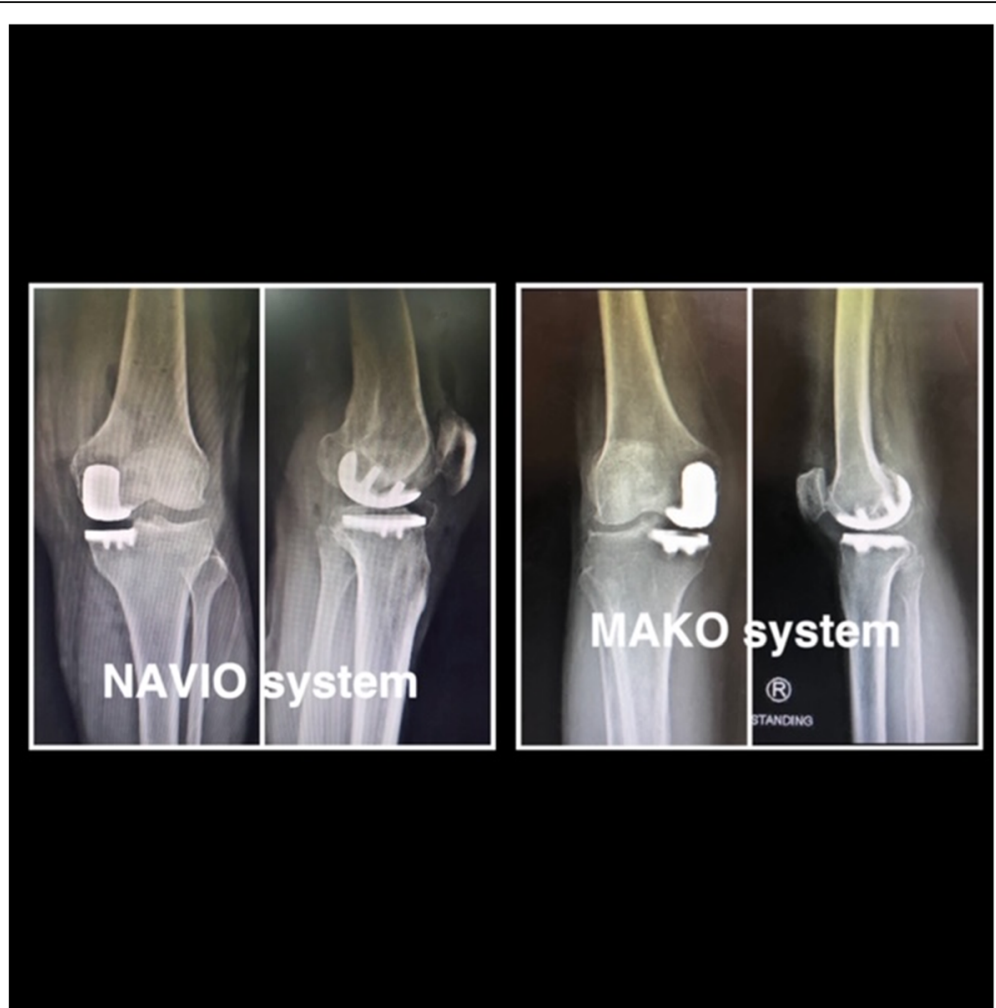

Fig. 2 X-rays of postoperative patient (our series) who underwent unicompartmental knee arthroplasty with image-based MAKO system and imageless NAVIO system. This picture show images of two patients who underwent two different procedure

subgroups and has a maximum score of 100 (the higher the score, the better the function). These were used by a well-trained research assistant (orthopedic resident and staff) at baseline and months $1,2,3,6$, and 12 after surgery. Any postoperative complications were recorded, such as deep vein thrombosis, infection, loosening of implants, fractures, lateral compartment arthritis, and dislocations of the polyethylene component.

\section{Radiological assessment}

Radiographic analysis of preoperative and postoperative images evaluating sagittal and coronal alignment and component positioning was performed by two fellowship-trained orthopedic surgeons with significant experience in UKA surgery. Postoperative femoral and tibial components were measured using radiographs 1 week and 12 months postoperatively, according to a previous report $[19,20]$. Inter- and intraobserver reliabilities were assessed using the kappa statistic.

\section{Statistical analysis}

The sample size was calculated to detect a mean difference in KSS (0-100) between MAKO and NAVIO robotassisted UKA. From the pilot study, the mean and standard deviation (SD) of KSSs in UKA with MAKO robotassisted and NAVIO robot-assisted UKA were 91.9 (5.0) and 89.6 (4.8), respectively. Type I error, power of test, and ratio of the treatment groups were set at $0.05,0.80$, and 1:1, respectively. The estimated sample size was 12 for each group in order to detect the minimal clinically significant mean difference of KSS of 6.1 units [21]. Loss to follow-up was estimated to be $20 \%$, which yields a required sample size of 15 patients per group.

Data were described using frequencies for categorical data and mean (SD) or median (range) as appropriate for continuous data. The baseline characteristics of the patients and cointerventions were compared between the two intervention groups using the chi-square test (or exact test when appropriate) and $t$ test for categorical data and continuous data, respectively. Continuous outcomes were the intraoperative time of seven steps, with KSS and KFS compared between intervention groups using a twosample $t$ test. Secondary analyses were done using a mixed linear regression analysis with a hierarchical approach. A $P$ value $<0.05$ was considered statistically significant. All analyses were performed using STATA version 15.0 software (StataCorp, College Station, TX, USA) [22].

\section{Results}

Patient characteristics

Clinical follow-up of 12 months was available in 33 knees (33 patients), comprising 16 NAVIO and 17 
MAKO robot-assisted UKA procedures (Fig. 1). There were no significant differences of preoperative demographics, clinical scores, and method of anesthesia (Table 1). All baseline characteristics were also comparable between treatment groups. Patient compliance with the allocated treatment and follow-up was $100 \%$ in both groups. Radiographic findings revealed no differences between the two groups (Tables 1 and 2).

\section{Seven steps of intraoperative time}

Seven steps of intraoperative time were plotted by treatment and time, which indicated that the MAKO group had faster time in all seven steps than the NAVIO group (Table 3). The mixed-effect regression model indicated that the MAKO group scored 14.55 (95\% confidence interval, 11.33, 17.77), significantly higher than the NAVIO group (Table 3).

\section{Mean Knee Society Score}

Mean KSS was plotted by treatment and time, which indicated increasing KSS after surgery in both groups. Applying the mixed-effect regression model indicated no significant difference between the two groups at each time point (Table 4).

\section{Mean Knee Functional Score}

Mean KFS was plotted by treatment and time, which indicated increasing KFS after surgery in both groups. Applying the mixed-effect regression model indicated significantly lower KFSs of 8.8 and 6.4 between the two groups at 1- and 2-month follow-up (Table 4). No perioperative or delayed complications (infection, periprosthetic fracture, thromboembolism, and compromised wound healing) and revisions were reported in either group.

\section{Discussion}

In this prospective cohort study, we compared the radiologic and clinical outcomes between imageless (NAVIO) and image-based (MAKO) robot-assisted UKA at 1 year. Our hypothesis was that use of the MAKO system in medial UKA could improve clinical outcomes, which include intraoperative (surgical time and blood loss) and postoperative outcomes, compared with use of the NAVIO surgical system, and this study showed no significant differences of KFS and KSS at 1 year. However, intraoperative time of seven steps (registration of hip and ankle, femur and tibia, ligament tension, implant planning, preparation femur, tibia, and trial implant) showed that the MAKO system required statistically significantly less time (about $16 \mathrm{~min}$ ) than the NAVIO system. In addition, intraoperative blood loss with the MAKO system was statistically significantly less by about $56 \mathrm{ml}$ than with the NAVIO system. No perioperative or delayed complications (infection, periprosthetic fracture, thromboembolism, and compromised wound healing) and revisions were reported in either group.

Our results show that the image-based system (MAKO) resulted in a significant decrease in operative time (registration to trial implant) and blood loss and an increase in knee function compared with the imageless system (NAVIO) in robot-assisted UKA. The reasons for this are undetermined in this study, but a possible explanation would be as follows. First, the MAKO robotic system uses an image-based procedure that is much faster than the NAVIO system, which may lead to less blood loss. Second, the MAKO system allows surgery to be tailored to the patient's anatomy, with more accurate reconstruction of the joint surfaces and the potential for more natural knee kinematics. Third, the use of a MAKO robotic arm-mounted irrigated burr rather than a traditional high-speed saw blade may prevent excessive heat-associated bone necrosis and might facilitate more

Table 2 Mean differences and 95\% confidence intervals of intraoperative time compared between NAVIO and MAKO robotically assisted unicompartmental knee arthroplasty

\begin{tabular}{|c|c|c|c|c|}
\hline Surgical steps & $\begin{array}{l}\text { NAVIO robotically assisted } \\
\text { UKA } \\
(n=16)\end{array}$ & $\begin{array}{l}\text { MAKO robotically assisted } \\
\text { UKA } \\
(n=17)\end{array}$ & $\begin{array}{l}\text { Mean difference and 95\% confidence } \\
\text { interval }\end{array}$ & $\begin{array}{l}P \\
\text { value }\end{array}$ \\
\hline Hip and ankle center registration & $5.6(3.9,7.2)$ & $2(0.6,3.4)$ & $3.6(1.5,5.7)$ & 0.001 \\
\hline Registration of femur and tibia & $8.4(6.8,10.0)$ & $5.4(4.0,6.8)$ & $3.0(0.9,5.1)$ & 0.005 \\
\hline Registration of ligament tension & $1.9(0.2,3.5)$ & $2.8(1.4,4.2)$ & $-0.9(-3.1,1.2)$ & 0.389 \\
\hline Implant planning & $6.1(4.5,7.7)$ & $5.2(3.8,6.5)$ & $0.9(-1.2,3.0)$ & 0.398 \\
\hline Femoral preparation & $11.5(9.9,13.1)$ & $7.6(6.2,9.0)$ & $3.9(1.8,6.0)$ & $\begin{array}{l}< \\
0.001\end{array}$ \\
\hline Tibial preparation & $8.2(6.6,9.8)$ & $8.0(6.6,9.4)$ & $0.2(-1.9,2.3)$ & 0.843 \\
\hline Trial implantation & $8.9(7.2,10.5)$ & $5.4(4.0,6.8)$ & $3.4(1.3,5.6)$ & 0.001 \\
\hline $\begin{array}{l}\text { Total operative time, min, mean } \\
\text { (SD) }\end{array}$ & $98.0(8.4)$ & $82.5(11.8)$ & $15.4(8.1,22.8)$ & 0.0002 \\
\hline
\end{tabular}

UKA unicompartmental knee arthroplasty 
Table 3 Mean Knee Society and Knee Functional scores compared between NAVIO and MAKO robotically assisted unicompartmental knee arthroplasty at follow-up time points 1, 2, 3, 6, and 12 months

\begin{tabular}{|c|c|c|c|c|}
\hline Follow-up time & $\begin{array}{l}\text { NAVIO robotically assisted UKA } \\
(n=16)\end{array}$ & $\begin{array}{l}\text { MAKO robotically assisted UKA } \\
(n=17)\end{array}$ & Mean difference and 95\% confidence interval & $P$ value \\
\hline \multicolumn{5}{|c|}{ Knee Society Score } \\
\hline 1 month & 91.9 & 89.6 & $2.2(-0.3,4.7)$ & 0.081 \\
\hline 2 months & 95.9 & 94.6 & $1.3(-1.2,3.8)$ & 0.313 \\
\hline 3 months & 97.3 & 96.4 & $0.8(-1.7,3.3)$ & 0.511 \\
\hline 6 months & 98.6 & 98.9 & $-0.32(-2.8,2.2)$ & 0.802 \\
\hline 12 months & 99.9 & 99.5 & $0.41(-2.1,2.9)$ & 0.749 \\
\hline \multicolumn{5}{|c|}{ Knee Functional Score } \\
\hline 1 month & 80.9 & 89.8 & $-8.8(-13.6,-4.0)$ & $<0.001$ \\
\hline 2 months & 86.3 & 92.6 & $-6.4(-11.2,-1.6)$ & 0.009 \\
\hline 3 months & 91.3 & 93.8 & $-2.6(-7.4,2.2)$ & 0.294 \\
\hline 6 months & 94.4 & 94.4 & $-0.04(-4.8,4.8)$ & 0.988 \\
\hline 12 months & 96.9 & 94.7 & $2.2(-2.6,7.0)$ & 0.376 \\
\hline
\end{tabular}

UKA unicompartmental knee arthroplasty

minimal bone resection, both of which may lead to less postoperative pain and blood loss and better function [16]. However, the differences of KFS at 1-2 months postoperatively were not clinically important, because clinical outcomes were similar with progression of time. As most patients still have pain, swelling, limited ROM, or related symptoms at 1-2 months after surgery, outcomes at this time may not reflect the final function of the patients.

Although MAKO and NAVIO robot-assisted UKA is not a new technique in arthroplasty and there have been many studies of MAKO or NAVIO robot-assisted UKA, there have been no studies comparing MAKO versus NAVIO robot-assisted UKA. To the best of our knowledge, this study is the first study that assessed any steps in operative time, KSS, KFS, and complications of UKA with MAKO compared with NAVIO robot-assisted UKA. The follow-up was reasonably high at a rate of $100 \%$ in both groups. We applied a subgroup analysis by adjusting unequal baseline characteristics of patients in both groups, thus minimizing bias.

\section{Limitations}

Our study has some limitations. First, the subjects in our study were not randomized into the two groups, because the baseline characteristics (severity of arthritis, preoperative KSS and KFS) would have been evaluated during surgery, and then the patients were included in either one group or another. However, we allocated the patients according to the date of surgery and blinded the evaluation of the intraoperative time, KSS, KFS, and complications of the patients in both groups. Second,

Table 4 Postoperative mechanical axis and mean value of each angle (coronal and sagittal of femur, tibia implant) in the two study groups

\begin{tabular}{|c|c|c|c|}
\hline Angle & $\begin{array}{l}\text { NAVIO robotically assisted UKA } \\
(n=16)\end{array}$ & $\begin{array}{l}\text { MAKO robotically assisted UKA } \\
(n=17)\end{array}$ & $P$ value \\
\hline \multicolumn{4}{|l|}{ Mechanical axis } \\
\hline Preoperative & $165.7(7.5)$ & $166.7(5.3)$ & 0.659 \\
\hline Postoperative & $180.1(2.2)$ & $179.7(1.5)$ & 0.544 \\
\hline \multicolumn{4}{|l|}{ Femoral } \\
\hline Coronal & $90.1(0.9)$ & $90.2(0.7)$ & 0.723 \\
\hline Sagittal & $90.2(1)$ & $89.7(0.4)$ & 0.066 \\
\hline \multicolumn{4}{|l|}{ Tibial } \\
\hline Coronal & $90(0.5)$ & $89.7(1)$ & 0.289 \\
\hline Sagittal & $-5.8(1)$ & $-6.1(1)$ & 0.396 \\
\hline
\end{tabular}

$<180$ of HKA (mechanical axis) and $<90$ of femoral, tibial coronal means varus

$<90$ of femoral sagittal means flexion

Negative value of tibial sagittal means postslope

UKA unicompartmental knee arthroplasty 
this is a small cohort with 16 patients in the NAVIO group and 17 patients in the MAKO group. Only 33 were eligible for statistical review. The sample size calculation was computed to assess primary outcomes between groups but may not be generalized to assess secondary outcomes; therefore, statistical insignificance might be due to the risk of type II error. Third, this study was done with a short follow-up period (shortterm [1-year] postoperative outcomes of UKA); thus, long-term effects of both techniques for UKA are still in question. Last, we considered only the KSS, KFS, and complication outcomes. Further study should assess outcomes such as costs of the operation and postoperative satisfaction and quality of life to compare MAKO and NAVIO robot-assisted UKA.

\section{Conclusion}

This study demonstrated that two robotic systems showed no differences in clinical outcomes at 1 year and radiologic alignment of implants, whereas operative time and intraoperative blood loss were found to be less in MAKO robot-assisted UKA.

\section{Acknowledgements}

All authors declare no funding source or sponsor involvement in the study design; collection, analysis, and interpretation of the data; in the writing of the manuscript; and in submission of the manuscript for publication.

\section{Authors' contributions \\ $\mathrm{CL}$ was responsible for study conception and design, collection and assembly of data, analysis and interpretation of the data, drafting of the manuscript, and final approval of the article. TT was responsible for study conception and design, collection and assembly of data, critical revision of the manuscript for important intellectual content, and final approval of the article. AA was responsible for manuscript writing, critical revision of the manuscript for important intellectual content, and final approval of the article. JK was responsible for study conception and design, collection and assembly of data, supervision of analysis and interpretation of the data, writing of the manuscript, critical revision of the manuscript for important intellectual content, and final approval of the article as well as statistical expertise. All authors read and approved the final manuscript.}

\section{Funding}

This study has no funding support.

\section{Availability of data and materials}

Not applicable.

\section{Ethics approval and consent to participate}

This study was approved by the Committee on Human Rights Related to Research Involving Human Subjects at the Bhumibol Adulyadej Hospital under protocol ID 2/62.

\section{Consent for publication}

Not applicable.

\section{Competing interests}

The authors declare that they have no competing interests.

\section{Author details}

'Orthopedics Department, Bhumibol Adulyadej Hospital, Bangkok, Thailand. ${ }^{2}$ Orthopedics Department, Bumrungrad Hospital, Bangkok, Thailand. ${ }^{3}$ Section for Clinical Epidemiology and Biostatistics, Faculty of Medicine, Ramathibodi
Hospital, Bangkok, Thailand. ${ }^{4}$ Orthopedic Department, Payathai 3 Hospital, Bangkok, Thailand.

Received: 22 October 2019 Accepted: 7 February 2020

Published online: 12 March 2020

\section{References}

1. Laurencin CT, Zelicof SB, Scott RD, Ewald FC (1991) Unicompartmental versus total knee arthroplasty in the same patient: a comparative study. Clin Orthop Relat Res 273:151-156

2. Patil S, Colwell CW Jr, Ezzet KA, D'Lima DD (2005) Can normal knee kinematics be restored with unicompartmental knee replacement? J Bone Joint Surg Am 87(2):332-338

3. Hollinghurst D, Stoney J, Ward T, Gill HS, Newman JH, Murray DW et al (2006) No deterioration of kinematics and cruciate function 10 years after medial unicompartmental arthroplasty. Knee 13(6):440-444

4. Lecuire F, Berard JB, Martres S (2014) Minimum 10-year follow-up results of ALPINA cementless hydroxyapatite-coated anatomic unicompartmental knee arthroplasty. Eur J Orthop Surg Traumatol 24(3):385-394

5. Lustig S, Elguindy A, Servien E, Fary C, Munini E, Demey G et al (2011) 5- to 16-year follow-up of 54 consecutive lateral unicondylar knee arthroplasties with a fixed-all polyethylene bearing. J Arthroplast 26(8):1318-1325

6. Hansen DC, Kusuma SK, Palmer RM, Harris KB (2014) Robotic guidance does not improve component position or short-term outcome in medial unicompartmental knee arthroplasty. J Arthroplasty 29(9):1784-1789

7. Bell SW, Anthony I, Jones B, MacLean A, Rowe P, Blyth M (2016) Improved accuracy of component positioning with robotic-assisted unicompartmental knee arthroplasty: data from a prospective, randomized controlled study. J Bone Joint Surg Am 98(8):627-635

8. Millar LJ, Banger M, Rowe PJ, Blyth M, Jones B, Maclean A (2018) O 017 - A five-year follow up of gait in robotic assisted vs conventional unicompartmental knee arthroplasty [abstract]. Gait Posture 65(Suppl 1):31-

9. Citak M, Suero EM, Citak M, Dunbar NJ, Branch SH, Conditt MA et al (2013) Unicompartmental knee arthroplasty: is robotic technology more accurate than conventional technique? Knee 20(4):268-271

10. Jenny JY, Ciobanu E, Boeri C (2007) The rationale for navigated minimally invasive unicompartmental knee replacement. Clin Orthop Relat Res 463: 58-62

11. Lonner JH (2009) Indications for unicompartmental knee arthroplasty and rationale for robotic arm-assisted technology. Am J Orthop (Belle Mead, NJ) 38(2 Suppl):3-6

12. Pearle AD, O'Loughlin PF, Kendoff DO (2010) Robot-assisted unicompartmental knee arthroplasty. J Arthroplast 25(2):230-237

13. Motesharei A, Rowe P, Blyth M, Jones B, Maclean A (2018) A comparison of gait one year post operation in an RCT of robotic UKA versus traditional Oxford UKA. Gait Posture 62:41-45

14. Canetti R, Batailler C, Bankhead C, Neyret P, Servien E, Lustig S (2018) Faster return to sport after robotic-assisted lateral unicompartmental knee arthroplasty: a comparative study. Arch Orthop Trauma Surg 138(12):17651771

15. Gilmour A, MacLean AD, Rowe PJ, Banger MS, Donnelly I, Jones BG et al (2018) Robotic-arm-assisted vs conventional unicompartmental knee arthroplasty: the 2-year clinical outcomes of a randomized controlled trial. J Arthroplasty 33(7S):S109-S115

16. Blyth MJG, Anthony I, Rowe P, Banger MS, MacLean A, Jones B (2017) Robotic arm-assisted versus conventional unicompartmental knee arthroplasty: exploratory secondary analysis of a randomised controlled trial. Bone Joint Res 6(11):631-639

17. Lonner JH, Klement MR (2019) Robotic-assisted medial unicompartmental knee arthroplasty: options and outcomes. J Am Acad Orthop Surg 27(5): e207-e214

18. Herry Y, Batailler C, Lording T, Servien E, Neyret P, Lustig S (2017) Improved joint-line restitution in unicompartmental knee arthroplasty using a roboticassisted surgical technique. Int Orthop 41(11):2265-2271

19. Hurst JM, Berend KR, Adams JB, Lombardi AV Jr (2015) Radiographic comparison of mobile-bearing partial knee single-peg versus twin-peg design. J Arthroplast 30(3):475-478

20. Kamenaga T, Hiranaka T, Nakanishi Y, Takayama K, Kuroda R, Matsumoto T (2019) Valgus subsidence of the tibial component caused by tibial 
component malpositioning in cementless oxford mobile-bearing unicompartmental knee arthroplasty. J Arthroplast 34(12):3054-3060

21. Lee WC, Kwan YH, Chong HC, Yeo SJ (2017) The minimal clinically important difference for Knee Society Clinical Rating System after total knee arthroplasty for primary osteoarthritis. Knee Surg Sports Traumatol Arthrosc 25(11):3354-3359

22. Chee-Kidd C, Vivek AS (2009) Is nonoperative treatment still indicated for Jones fracture? Eur J Trauma Emerg Surg 35(4):407 Published correction appears in Eur J Trauma Emerg Surg. 2010;36(3):270

\section{Publisher's Note}

Springer Nature remains neutral with regard to jurisdictional claims in published maps and institutional affiliations.

Ready to submit your research? Choose BMC and benefit from:

- fast, convenient online submission

- thorough peer review by experienced researchers in your field

- rapid publication on acceptance

- support for research data, including large and complex data types

- gold Open Access which fosters wider collaboration and increased citations

- maximum visibility for your research: over $100 \mathrm{M}$ website views per year

At $B M C$, research is always in progress.

Learn more biomedcentral.com/submissions 